\title{
$p$-adic singular integrals and their commutators in generalized Morrey spaces
}

Hui X. Mo ${ }^{1 *}$ (O), Zhe Han', Liu Yang' and Xiao J. Wang'

"Correspondence:

huixmo@bupt.edu.cn

'School of Science, Beijing

University of Posts and

Telecommunications, Beijing, China

\begin{abstract}
For a prime number $p$, let $\mathbb{Q}_{p}$ be the field of $p$-adic numbers. In this paper, we establish the boundedness of a class of $p$-adic singular integral operators on the $p$-adic generalized Morrey spaces. We also consider the corresponding boundedness for the commutators generalized by the $p$-adic singular integral operators and $p$-adic Lipschitz functions or $p$-adic generalized Campanato functions.
\end{abstract}

MSC: 42B20; 42B25

Keywords: $p$-adic field; $p$-adic singular integral operator; Commutator; $p$-adic generalized Morrey function; $p$-adic generalized Campanato function; $p$-adic Lipschitz function

\section{Introduction}

Let $p$ be a prime number, and let $x \in \mathbb{Q}$. Then the non-Archimedean $p$-adic norm $|x|_{p}$ is defined as follows: if $x=0$, then $|0|_{p}=0$; if $x \neq 0$ is an arbitrary rational number with unique representation $x=p^{\gamma} \frac{m}{n}$, where $m, n$ are not divisible by $p$, and $\gamma=\gamma(x) \in \mathbb{Z}$, then $|x|_{p}=p^{-\gamma}$. This norm has the following properties: $|x y|_{p}=|x|_{p}|y|_{p},|x+y|_{p} \leq \max \left\{|x|_{p},|y|_{p}\right\}$, and $|x|_{p}=0$ if and only if $x=0$. Moreover, when $|x|_{p} \neq|y|_{p}$, we have $|x+y|_{p}=\max \left\{|x|_{p},|y|_{p}\right\}$. Let $\mathbb{Q}_{p}$ be the field of $p$-adic numbers defined as the completion of the field of rational numbers $\mathbb{Q}$ with respect to the non-Archimedean $p$-adic norm $|\cdot|_{p}$. For $\gamma \in \mathbb{Z}$, we denote the ball $B_{\gamma}(a)$ with center at $a \in \mathbb{Q}_{p}$ and radius $p^{\gamma}$ and its boundary $S_{\gamma}(a)$ by

$$
B_{\gamma}(a)=\left\{x \in \mathbb{Q}_{p}:|x-a|_{p} \leq p^{\gamma}\right\}, \quad S_{\gamma}(a)=\left\{x \in \mathbb{Q}_{p}:|x-a|_{p}=p^{\gamma}\right\},
$$

respectively. It is easy to see that

$$
B_{\gamma}(a)=\bigcup_{k \leq \gamma} S_{k}(a)
$$

For $n \in \mathbb{N}$, the space $\mathbb{Q}_{p}^{n}=\mathbb{Q}_{p} \times \cdots \times \mathbb{Q}_{p}$ consists of all points $x=\left(x_{1}, \ldots, x_{n}\right)$ where $x_{i} \in \mathbb{Q}_{p}, i=1, \ldots, n, n \geq 1$. The $p$-adic norm of $\mathbb{Q}_{p}^{n}$ is defined by

$$
|x|_{p}=\max _{1 \leq i \leq n}\left|x_{i}\right|_{p}, \quad x \in \mathbb{Q}_{p}^{n} .
$$

(c) The Author(s) 2019. This article is distributed under the terms of the Creative Commons Attribution 4.0 International License (http://creativecommons.org/licenses/by/4.0/), which permits unrestricted use, distribution, and reproduction in any medium, provided you give appropriate credit to the original author(s) and the source, provide a link to the Creative Commons license, and indicate if changes were made. 
Thus it is easy to see that $|x|_{p}$ is a non-Archimedean norm on $\mathbb{Q}_{p}^{n}$. The balls $B_{\gamma}(a)$ and the sphere $S_{\gamma}(a)$ in $\mathbb{Q}_{p}^{n}$ for $\gamma \in \mathbb{Z}$ are defined similarly to the case $n=1$.

Since $\mathbb{Q}_{p}^{n}$ is a locally compact commutative group under addition, by the standard analysis there exists the Haar measure $d x$ on the additive group $\mathbb{Q}_{p}^{n}$ normalized by $\int_{B_{0}} d x=$ $\left|B_{0}\right|_{H}=1$, where $|E|_{H}$ denotes the Haar measure of a measurable set $E \subset \mathbb{Q}_{p}^{n}$. Then by a simple calculation the Haar measures of any balls and spheres can be obtained. From the integral theory it is easy to see that $\left|B_{\gamma}(a)\right|_{H}=p^{n \gamma}$ and $\left|S_{\gamma}(a)\right|_{H}=p^{n \gamma}\left(1-p^{-n}\right)$ for any $a \in \mathbb{Q}_{p}^{n}$. For a more complete introduction to the $p$-adic analysis, we refer to [1-8] and the references therein.

The $p$-adic numbers have been applied in the string theory, turbulence theory, statistical mechanics, quantum mechanics, and so forth (see $[1,9,10]$ for detail). In the past few years, there is an increasing interest in the study of harmonic analysis on $p$-adic field (see [5-8] for detail).

Let $\Omega \in L^{\infty}\left(\mathbb{Q}_{p}^{n}\right)$ be such that $\Omega\left(p^{j} x\right)=\Omega(x)$ for all $j \in \mathbb{Z}$ and $\int_{|x|_{p}=1} \Omega(x) d x=0$. Then the $p$-adic singular integral operators defined by Taibleson [5] are as follows:

$$
T_{k}(f)(x)=\int_{|y|_{p}>p^{k}} f(x-y) \frac{\Omega(y)}{|y|_{p}^{n}} d z \quad \text { for } k \in \mathbb{Z} .
$$

The $p$-adic singular integral operator $T$ is defined as the limit of $T_{k}$ as $k$ goes to $-\infty$.

Moreover, let $\vec{b}=\left(b_{1}, b_{2}, \ldots, b_{m}\right)$, where $b_{i} \in L_{\mathrm{loc}}\left(\mathbb{Q}_{p}^{n}\right)$ for $1 \leq i \leq m$. Then the higher commutator generated by $\vec{b}$ and $T_{k}$ can be defined as

$$
T_{k}^{\vec{b}} f(x)=\int_{|y| p>p^{k}} \prod_{i=1}^{m}\left(b_{i}(x)-b_{i}(x-y)\right) f(x-y) \frac{\Omega(y)}{|y|_{p}^{n}} d z \quad \text { for } k \in \mathbb{Z}
$$

and the commutator generated by $\vec{b}=\left(b_{1}, b_{2}, \ldots, b_{m}\right)$ and the $p$-adic singular integral operator $T$ is defined as the limit of $T_{k}^{\vec{b}}$ as $k$ goes to $-\infty$.

Under some conditions, the authors in $[5,11]$ showed that $T_{k}$ were of type $(q, q)$ for $1<q<\infty$ and of weak type $(1,1)$ on local fields. Wu et al. [12] established the boundedness of $T_{k}$ on $p$-adic central Morrey spaces. Furthermore, the $\lambda$-central BMO estimates for commutators of these singular integral operators on $p$-adic central Morrey spaces were obtained in [12]. Moreover, in the $p$-adic linear space $\mathbb{Q}_{p}^{n}$, Volosivets [13] gave sufficient conditions for the boundedness of the maximal function and Riesz potential in $p$-adic generalized Morrey spaces. Mo et al. [14] established the boundedness of the commutators generated by the $p$-adic Riesz potential and $p$-adic generalized Campanato functions in $p$ adic generalized Morrey spaces.

Motivated by the works of [12-14], we consider the boundedness of $T_{k}$ on the $p$-adic generalized Morrey type spaces, as well as the boundedness of the commutators generated by $T_{k}$ and $p$-adic generalized Campanato functions.

Throughout this paper, the letter $C$ will be used to denote constants varying from line to line. The relation $A \lesssim B$ means that $A \leq C B$ with some positive constant $C$ independent of appropriate quantities.

\section{Some notation and lemmas}

Definition 2.1 ([13]) Let $1 \leq q<\infty$, and let $\omega(x)$ be a nonnegative measurable function in $\mathbb{Q}_{p}^{n}$. A function $f \in L_{\text {loc }}^{q}\left(\mathbb{Q}_{p}^{n}\right)$ is said to belong to the generalized Morrey space $G M_{q, \omega}\left(\mathbb{Q}_{p}^{n}\right)$ 
if

$$
\|f\|_{G M_{q, \omega}}=\sup _{a \in \mathbb{Q}_{p}^{n}, \gamma \in \mathbb{Z}} \frac{1}{\omega\left(B_{\gamma}(a)\right)}\left(\frac{1}{\left|B_{\gamma}(a)\right|_{H}} \int_{B_{\gamma}(a)}|f(y)|^{q} d y\right)^{1 / q}<\infty
$$

where $\omega\left(B_{\gamma}(a)\right)=\int_{B_{\gamma}(a)} \omega(x) d x$.

Let $\lambda \in \mathbb{R}$. If $\omega\left(B_{\gamma}(a)\right)=\left|B_{\gamma}(a)\right|^{\lambda}$, then $G M_{q, \omega}\left(\mathbb{Q}_{p}^{n}\right)$ is the classical Morrey space $M_{q, \lambda}\left(\mathbb{Q}_{p}^{n}\right)$. About the generalized Morrey space, see [15], and for the classical Morrey spaces, see [16] and so on.

Moreover, let $\lambda \in \mathbb{R}$ and $1 \leq q<\infty$. The $p$-adic central Morrey space $C M_{q, \lambda}\left(\mathbb{Q}_{p}^{n}\right)$ (see [8]) is defined by

$$
\|f\|_{C M_{q, \lambda}}=\sup _{\gamma \in \mathbb{Z}}\left(\frac{1}{\left|B_{\gamma}(0)\right|_{H}^{1+\lambda q}} \int_{B_{\gamma}(0)}|f(y)|^{q} d y\right)^{1 / q}<\infty .
$$

Definition 2.2 ([17]) For $0<\beta<1$, the the $p$-adic Lipschitz space $\Lambda_{\beta}\left(\mathbb{Q}_{p}^{n}\right)$ is defined as the set of all functions $f: \mathbb{Q}_{p}^{n} \mapsto \mathbb{C}$ such that

$$
\|f\|_{\Lambda_{\beta}\left(\mathbb{Q}_{p}^{n}\right)}=\sup _{x, h \in \mathbb{Q}_{p}^{n}, h \neq 0} \frac{|f(x+h)-f(x)|}{|h|_{p}^{\beta}}<\infty .
$$

Definition 2.3 ([13]) Let $B$ be a ball in $\mathbb{Q}_{p}^{n}, 1 \leq q<\infty$, and let $\omega(x)$ be a nonnegative measurable function in $\mathbb{Q}_{p}^{n}$. A function $f \in L_{\text {loc }}^{q}\left(\mathbb{Q}_{p}^{n}\right)$ is said to belong to the generalized Campanato space $G C_{q, \omega}\left(\mathbb{Q}_{p}^{n}\right)$ if

$$
\|f\|_{G C_{q, \omega}}=\sup _{a \in \mathbb{Q}_{p}^{n}, \gamma \in \mathbb{Z}} \frac{1}{\omega\left(B_{\gamma}(a)\right)}\left(\frac{1}{\left|B_{\gamma}(a)\right|_{H}} \int_{B_{\gamma}(a)}\left|f(y)-f_{B_{\gamma}(a)}\right|^{q} d y\right)^{1 / q}<\infty,
$$

where $f_{B_{\gamma}(a)}=\frac{1}{\left|B_{\gamma}(a)\right|_{H}} \int_{B_{\gamma}(a)} f(x) d x$ and $\omega\left(B_{\gamma}(a)\right)=\int_{B_{\gamma}(a)} \omega(x) d x$.

The classical Campanato spaces can be found in [18, 19], and so on. The important particular case of $G C_{q, \omega}\left(\mathbb{Q}_{p}^{n}\right)$ is $B M O_{q, \lambda}\left(\mathbb{Q}_{p}^{n}\right)$, where $1<q<\infty$ and $0<\lambda<1 / n$. The central BMO space $C B M O_{q, \lambda}\left(\mathbb{Q}_{p}^{n}\right)$ is defined by

$$
\|f\|_{C B M O,, \lambda\left(\mathbb{Q}_{p}^{n}\right)}=\sup _{\gamma \in \mathbb{Z}} \frac{1}{\left|B_{\gamma}(0)\right|_{H}^{\lambda}}\left(\frac{1}{\left|B_{\gamma}(0)\right|_{H}} \int_{B_{\gamma}(0)}\left|f(y)-f_{B_{\gamma}(0)}\right|^{q} d y\right)^{1 / q}<\infty .
$$

Lemma 2.1 ([14]) Let $1 \leq q<\infty$, and let $\omega$ be a nonnegative measurable function. Let $b \in G C_{q, \omega}\left(\mathbb{Q}_{p}^{n}\right)$. Then

$$
\left|b_{B_{k}(a)}-b_{B_{j}(a)}\right| \leq\|b\|_{G C_{q, \omega}}|j-k| \max \left\{\omega\left(B_{k}(a)\right), \omega\left(B_{j}(a)\right)\right\}
$$

for $j, k \in \mathbb{Z}$ and any fixed $a \in \mathbb{Q}_{p}^{n}$.

Thus, for $j>k$, from Lemma 2.1 we deduce that

$$
\left(\int_{B_{j}(a)}\left|b(y)-b_{B_{k}(a)}\right|^{q} d y\right)^{1 / q} \leq(j+1-k)\left|B_{j}(a)\right|_{H}^{1 / q} \omega\left(B_{j}(a)\right)\|b\|_{G C_{q, \omega}} .
$$


Lemma 2.2 ([5]) Let $\Omega \in L^{\infty}\left(\mathbb{Q}_{p}^{n}\right)$ be such that $\Omega\left(p^{j} x\right)=\Omega(x)$ for all $j \in \mathbb{Z}$ and $\int_{|x|_{p}=1} \Omega(x) d x=0$. If

$$
\sup _{|y|_{p}=1} \sum_{j=1}^{\infty} \int_{|x|_{p}=1}\left|\Omega\left(x+p^{j} y\right)-\Omega(x)\right| d x<\infty
$$

then for $1<p<\infty$, there is a constant $C>0$ such that

$$
\left\|T_{k}(f)\right\|_{L^{p}\left(\mathbb{Q}_{p}^{n}\right)} \leq C\|f\|_{L^{p}\left(\mathbb{Q}_{p}^{n}\right)}
$$

for $k \in \mathbb{Z}$, where $C$ is independent off and $k \in \mathbb{Z}$.

Furthermore, $T(f)=\lim _{k \rightarrow-\infty} T_{k}(f)$ exists in the $L^{p}$ norm, and

$$
\|T(f)\|_{L^{p}\left(\mathbb{Q}_{p}^{n}\right)} \leq C\|f\|_{L^{p}\left(\mathbb{Q}_{p}^{n}\right)}
$$

Moreover, on the $p$-adic field, the Riesz potential $I_{\alpha}^{p}$ is defined by

$$
I_{p}^{\alpha} f(x)=\frac{1}{\Gamma_{n}(\alpha)} \int_{\mathbb{Q}_{p}^{n}} \frac{f(y)}{|x-y|_{p}^{n-\alpha}} d y,
$$

where $\Gamma_{n}(\alpha)=\left(1-p^{\alpha-n}\right) /\left(1-p^{-\alpha}\right)$ for $\alpha \in \mathbb{C}, \alpha \neq 0$.

Lemma 2.3 ([14]) Let $\alpha$ be a complex number with $0<\operatorname{Re} \alpha<n$, and let $1<r<\infty, 1<q<$ $n / \operatorname{Re} \alpha$, and $0<1 / r=1 / q-\operatorname{Re} \alpha / n$. Suppose that both $\omega$ and $v$ are nonnegative measurable functions such that

$$
\sum_{j=\gamma}^{\infty} p^{j \operatorname{Re} \alpha} \frac{\nu\left(B_{j}(a)\right)}{\omega\left(B_{\gamma}(a)\right)}=C<\infty
$$

for any $a \in \mathbb{Q}_{p}^{n}$ and $\gamma \in \mathbb{Z}$. Then the Riesz potential $I_{p}^{\alpha}$ is bounded from $G M_{q, v}$ to $G M_{r, \omega}$.

\section{Main results}

In this section, we state the main results of the paper.

Theorem 3.1 Let $1<q<\infty$, and let $\Omega\left(p^{j} x\right)=\Omega(x)$ for all $j \in \mathbb{Z}, \int_{|x|_{p}=1} \Omega(x) d x=0$, and

$$
\sup _{|y|_{p}=1} \sum_{j=1}^{\infty} \int_{|x|_{p}=1}\left|\Omega\left(x+p^{j} y\right)-\Omega(x)\right| d x<\infty
$$

Suppose that both $\omega$ and $v$ are nonnegative measurable functions such that

$$
\sum_{j=\gamma}^{\infty} v\left(B_{j}(a)\right) / \omega\left(B_{\gamma}(a)\right)=C<\infty
$$

for any $\gamma \in \mathbb{Z}$ and $a \in \mathbb{Q}_{p}^{n}$. Then the singular integral operators $T_{k}$ are bounded from $G M_{q, v}$ to $G M_{q, \omega}$ for all $k \in \mathbb{Z}$. Moreover, $T(f)=\lim _{k \rightarrow-\infty} T_{k}(f)$ exists in $G M_{q, \omega}$, and the operator $T$ is bounded from $G M_{q, v}$ to $G M_{q, \omega}$. 
Corollary 3.1 Let $1<q<\infty, \lambda<0$, and let $\Omega \in L^{\infty}\left(\mathbb{Q}_{p}^{n}\right)$ be such that $\Omega\left(p^{j} x\right)=\Omega(x)$ for all $j \in \mathbb{Z}, \int_{|x| p=1} \Omega(x) d x=0$, and

$$
\sup _{|y| p=1} \sum_{j=1}^{\infty} \int_{|x|_{p}=1}\left|\Omega\left(x+p^{j} y\right)-\Omega(x)\right| d x<\infty
$$

Then the operators $T_{k}$ and $T$ are bounded on the space $M_{q, \lambda}$ for all $k \in \mathbb{Z}$.

In fact, for $\lambda<0$, taking $\omega(B)=v(B)=|B|_{H}^{\lambda}$ in Theorem 3.1, we obtain Corollary 3.1. If the Morrey space $M_{q, \lambda}\left(\mathbb{Q}_{p}^{n}\right)$ is replaced by the central Morrey space $C M_{q, \lambda}\left(\mathbb{Q}_{p}^{n}\right)$ in Corollary 3.1, then the conclusion is that of Theorem 4.1 in [12].

Theorem 3.2 Let $\Omega \in L^{\infty}\left(\mathbb{Q}_{p}^{n}\right)$ be such that $\Omega\left(p^{j} x\right)=\Omega(x)$ for all $j \in \mathbb{Z}, \int_{|x|_{p}=1} \Omega(x) d x=0$, and

$$
\sup _{|y|_{p}=1} \sum_{j=1}^{\infty} \int_{|x|_{p}=1}\left|\Omega\left(x+p^{j} y\right)-\Omega(x)\right| d x<\infty
$$

Let $0<\beta_{i}<1$ for $i=1,2, \ldots, m$ be such that $0<\beta=\sum_{i=1}^{m} \beta_{i}<n$, and let $1<r<\infty$ and $1<q<n / \beta$ be such that $1 / r=1 / q-\beta / n$. Suppose that $b_{i} \in \Lambda_{\beta_{i}}, i=1,2, \ldots, m$, and both $\omega$ and $v$ are nonnegative measurable functions such that

$$
\sum_{j=\gamma}^{\infty} p^{j \beta} v\left(B_{j}(a)\right) / \omega\left(B_{\gamma}(a)\right)=C<\infty
$$

for any $\gamma \in \mathbb{Z}$ and $a \in \mathbb{Q}_{p}^{n}$. Then the commutators $T_{k}^{\vec{b}}$ are bounded from $G M_{q, v}$ to $G M_{r, \omega}$ for all $k \in \mathbb{Z}$. Moreover, the commutator $T^{\vec{b}}(f)=\lim _{k \rightarrow-\infty} T_{k}^{\vec{b}}(f)$ exists in the space of $G M_{q, \omega}$, and $T^{\vec{b}}$ is bounded from $G M_{q, v}$ to $G M_{q, \omega}$.

Theorem 3.3 Let $\Omega \in L^{\infty}\left(\mathbb{Q}_{p}^{n}\right)$ be such that $\Omega\left(p^{j} x\right)=\Omega(x)$ for all $j \in \mathbb{Z}, \int_{|x|_{p}=1} \Omega(x) d x=0$, and

$$
\sup _{|y|_{p}=1} \sum_{j=1}^{\infty} \int_{|x|_{p}=1}\left|\Omega\left(x+p^{j} y\right)-\Omega(x)\right| d x<\infty
$$

Let $1<q, r, q_{1}, \ldots, q_{m}<\infty$ be such that $1 / r=1 / q+1 / q_{1}+1 / q_{2}+\cdots+1 / q_{m}$. Suppose that $\omega, v$, and $v_{i}(i=1,2, \ldots, m)$ are nonnegative measurable functions. Suppose that $b_{i} \in G C_{q_{i}, v_{i}}\left(\mathbb{Q}_{p}^{n}\right)$, $i=1,2, \ldots, m$, and the functions $\omega, v$, and $v_{i}(i=1,2, \ldots, m)$ satisfy the following conditions:

(i) $\prod_{i=1}^{m} v_{i}\left(B_{\gamma}(a)\right) v\left(B_{\gamma}(a)\right) / \omega\left(B_{\gamma}(a)\right)=C<\infty$,

(ii) $\sum_{j=\gamma+1}^{\infty} \prod_{i=1}^{m} v_{i}\left(B_{j}(a)\right)(j+1-\gamma)^{m} v\left(B_{j}(a)\right) / \omega\left(B_{\gamma}(a)\right)=C<\infty$

for any $\gamma \in \mathbb{Z}$ and $a \in \mathbb{Q}_{p}^{n}$. Then the commutators $T_{k}^{\vec{b}}$ are bounded from $G M_{q, v}$ to $G M_{r, \omega}$ for all $k \in \mathbb{Z}$. The commutator $T^{\vec{b}}=\lim _{k \rightarrow-\infty} T_{k}^{\vec{b}}$ exists in the space of $G M_{q, \omega}$, and $T^{\vec{b}}$ is bounded from $G M_{q, v}$ to $G M_{q, \omega}$. 
Corollary 3.2 Let $\Omega \in L^{\infty}\left(\mathbb{Q}_{p}^{n}\right)$ be such that $\Omega\left(p^{j} x\right)=\Omega(x)$ for all $j \in \mathbb{Z}, \int_{|x|_{p}=1} \Omega(x) d x=0$, and

$$
\sup _{|y|_{p}=1} \sum_{j=1}^{\infty} \int_{|x|_{p}=1}\left|\Omega\left(x+p^{j} y\right)-\Omega(x)\right| d x<\infty
$$

Let $1<q, r, q_{1}, \ldots, q_{m}<\infty$ be such that $1 / r=1 / q+1 / q_{1}+1 / q_{2}+\cdots+1 / q_{m}$. Let $0 \leq$ $\lambda_{1}, \ldots, \lambda_{m}<1 / n, \lambda<-\sum_{i=1}^{m} \lambda_{i}$, and $\tilde{\lambda}=\sum_{i=1}^{m} \lambda_{i}+\lambda$. If $b_{i} \in B M O_{q_{i}, \lambda_{i}}\left(\mathbb{Q}_{p}^{n}\right)$, then the commutators $T_{k}^{\vec{b}}$ and $T^{\vec{b}}$ are bounded from $M_{q, \lambda}$ to $M_{r, \tilde{\lambda}}$ for all $k \in \mathbb{Z}$.

Moreover, let $1<r, q, q_{1}<\infty$ be such that $1 / r=1 / q+1 / q_{1}$. Let $0 \leq \lambda_{1}<1 / n, \lambda<-\lambda_{1}$, and $\tilde{\lambda}=\lambda_{1}+\lambda$. If $b \in C B M O_{q_{1}, \lambda_{1}}\left(\mathbb{Q}_{p}^{n}\right)$, then from Corollary 3.1 it follows that the commutators $T_{k}^{b}=\left[T_{k}, b\right]$ and $T^{b}=[T, b]$ are bounded from $C M_{q, \lambda}$ to $C M_{r, \tilde{\lambda}}$ for all $k \in \mathbb{Z}$. These results are those of Theorem 4.2 in [12].

\section{Proof of Theorems 3.1-3.3}

Let us first give the proof of Theorem 3.1.

For any fixed $\gamma \in \mathbb{Z}$ and $a \in \mathbb{Q}_{p}^{n}$, it is easy to see that

$$
\begin{aligned}
\frac{1}{\omega\left(B_{\gamma}(a)\right)}\left(\frac{1}{\left|B_{\gamma}(a)\right|_{H}} \int_{B_{\gamma}(a)}\left|T_{k}(f)(x)\right|^{q} d x\right)^{1 / q} \\
\leq \frac{1}{\omega\left(B_{\gamma}(a)\right)}\left(\frac{1}{\left|B_{\gamma}(a)\right|_{H}} \int_{B_{\gamma}(a)}\left|T_{k}(f)\left(f \chi_{B_{\gamma}(a)}\right)(x)\right|^{q} d x\right)^{1 / q} \\
\quad+\frac{1}{\omega\left(B_{\gamma}(a)\right)}\left(\frac{1}{\left|B_{\gamma}(a)\right|_{H}} \int_{B_{\gamma}(a)}\left|T_{k}\left(f \chi_{B_{\gamma}^{c}(a)}\right)(x)\right|^{q} d x\right)^{1 / q} \\
:=I+I I,
\end{aligned}
$$

where $B_{\gamma}^{c}(a)$ is the complement to $B_{\gamma}(a)$ in $\mathbb{Q}_{p}^{n}$.

Using Lemma 2.2 and (3.1), it follows that

$$
\begin{aligned}
I & \lesssim \frac{1}{\omega\left(B_{\gamma}(a)\right)} \frac{1}{\left|B_{\gamma}(a)\right|_{H}^{1 / q}}\left(\int_{B_{\gamma}(a)}|f(x)|^{q} d x\right)^{1 / q} \\
& =\frac{\nu\left(B_{\gamma}(a)\right)}{\omega\left(B_{\gamma}(a)\right)} \frac{1}{v\left(B_{\gamma}(a)\right)}\left(\frac{1}{\left|B_{\gamma}(a)\right|_{H}} \int_{B_{\gamma}(a)}|f(x)|^{q} d x\right)^{1 / q} \\
& \lesssim\|f\|_{G M_{q, v}} .
\end{aligned}
$$

For $I I$, let us first estimate $\left|T_{k}\left(f \chi_{B_{\gamma}^{c}(a)}\right)(x)\right|$.

Since $x \in B_{\gamma}(a)$ and $\Omega \in L^{\infty}\left(\mathbb{Q}_{p}^{n}\right)$, we have

$$
\begin{aligned}
\left|T_{k}\left(f \chi_{B_{\gamma}^{c}(a)}\right)(x)\right| & =\left|\int_{|y|_{p}>p^{k}}\left(f \chi_{B_{\gamma}^{c}(a)}\right)(x-y) \frac{\Omega(y)}{|y|_{p}^{n}} d y\right| \\
& =\left|\int_{|x-z| p>p^{k}}\left(f \chi_{B_{\gamma}^{c}(a)}\right)(z) \frac{\Omega(x-z)}{|x-z|_{p}^{n}} d z\right| \\
& \lesssim \int_{B_{\gamma}^{c}(a)} \frac{|f(z)|}{|x-z|_{p}^{n}} d z
\end{aligned}
$$




$$
\begin{aligned}
& \lesssim \sum_{j=\gamma+1}^{\infty} \int_{S_{j}(a)} p^{-j n}|f(y)| d y \\
& \leq \sum_{j=\gamma+1}^{\infty} p^{-j n}\left(\int_{B_{j}(a)}|f(y)|^{q} d y\right)^{1 / q}\left|B_{j}(a)\right|_{H}^{1-1 / q} \\
& =\|f\|_{G M_{q, v}} \sum_{j=\gamma+1}^{\infty} v\left(B_{j}(a)\right) .
\end{aligned}
$$

Thus from (3.1) and (4.3) it follows that

$$
\begin{aligned}
I I & =\frac{1}{\omega\left(B_{\gamma}(a)\right)}\left(\frac{1}{\left|B_{\gamma}(a)\right|_{H}} \int_{B_{\gamma}(a)}\left|T_{k}\left(f \chi_{B_{\gamma}^{c}(a)}\right)(x)\right|^{q} d x\right)^{1 / q} \\
& \lesssim\|f\|_{G M_{q, v}} \sum_{j=\gamma+1}^{\infty} v\left(B_{j}(a)\right) / \omega\left(B_{\gamma}(a)\right) \\
& \lesssim\|f\|_{G M_{q, v}} .
\end{aligned}
$$

Combining the estimates of (4.1), (4.2), and (4.4), we have

$$
\frac{1}{\omega\left(B_{\gamma}(a)\right)}\left(\frac{1}{\left|B_{\gamma}(a)\right|_{H}} \int_{B_{\gamma}(a)}\left|T_{k}(f)(x)\right|^{q} d x\right)^{1 / q} \lesssim\|f\|_{G M_{q, v}},
$$

which means that $T_{k}$ is bounded from $G M_{q, v}$ to $G M_{q, \omega}$ for all $k \in \mathbb{Z}$.

Moreover, from Lemma 2.2 and the definition of $G M_{q, \omega}\left(\mathbb{Q}_{p}^{n}\right)$ it is obvious that $T(f)=$ $\lim _{k \rightarrow-\infty} T_{k}(f)$ exists in $G M_{q, \omega}$ and the operator $T$ is bounded from $G M_{q, v}$ to $G M_{q, \omega}$.

Proof of Theorem 3.2 For any $x \in \mathbb{Q}_{p}^{n}$, since $\Omega \in L^{\infty}\left(\mathbb{Q}_{p}^{n}\right)$ and $b_{i} \in \Lambda_{\beta_{i}}, i=1,2, \ldots, m$, it is easy to see that

$$
\begin{aligned}
& \left|T_{k}^{\vec{b}} f(x)\right| \\
& \quad \leq \int_{|y| p>p^{k}} \prod_{i=1}^{m}\left|b_{i}(x)-b_{i}(x-y)\right||f(x-y)| \frac{|\Omega(y)|}{|y|_{p}^{n}} d y \\
& \quad \lesssim \int_{\mathbb{Q}_{p}^{n}} \frac{|f(z)|}{|x-z|_{p}^{n-\beta}} d z \\
& \quad \lesssim I_{p}^{\beta}(|f|)(x) .
\end{aligned}
$$

Thus from Lemma 2.3 it is obvious that the commutators $T_{k}^{\vec{b}}$ are bounded from $G M_{q, v}$ to $G M_{r, \omega}$ for all $k \in \mathbb{Z}$.

Moreover, from the definition of $G M_{q, \omega}\left(\mathbb{Q}_{p}^{n}\right)$ it is obvious that $T^{\vec{b}}(f)=\lim _{k \rightarrow-\infty} T_{k}^{\vec{b}}(f)$ exists in the space of $G M_{q, \omega}$, and the commutator $T^{\vec{b}}$ is bounded from $G M_{q, v}$ to $G M_{q, \omega}$.

Proof of Theorem 3.3 Without loss of generality, we need only to show that the conclusion holds for $m=2$. 
For any fixed $\gamma \in \mathbb{Z}$ and $a \in \mathbb{Q}_{p}^{n}$, we write $f^{0}=f \chi_{B_{\gamma}(a)}$ and $f^{\infty}=f \chi_{B_{\gamma}^{c}(a)}$. Then

$$
\begin{aligned}
& \frac{1}{\omega\left(B_{\gamma}(a)\right)}\left(\frac{1}{\left|B_{\gamma}(a)\right|_{H}} \int_{B_{\gamma}(a)}\left|T_{k}^{\left(b_{1}, b_{2}\right)}(f)(x)\right|^{r} d x\right)^{1 / r} \\
& \leq \frac{1}{\omega\left(B_{\gamma}(a)\right)}\left(\frac{1}{\left|B_{\gamma}(a)\right|_{H}} \int_{B_{\gamma}(a)}\left|\left(b_{1}(x)-\left(b_{1}\right)_{B_{\gamma}(a)}\right)\left(b_{2}(x)-\left(b_{2}\right)_{B_{\gamma}(a)}\right) T_{k}\left(f^{0}\right)(x)\right|^{r} d x\right)^{1 / r} \\
& +\frac{1}{\omega\left(B_{\gamma}(a)\right)}\left(\frac{1}{\left|B_{\gamma}(a)\right|_{H}} \int_{B_{\gamma}(a)}\left|\left(b_{1}(x)-\left(b_{1}\right)_{B_{\gamma}(a)}\right) T_{k}\left(\left(b_{2}-\left(b_{2}\right)_{B_{\gamma}(a)}\right) f^{0}\right)(x)\right|^{r} d x\right)^{1 / r} \\
& +\frac{1}{\omega\left(B_{\gamma}(a)\right)}\left(\frac{1}{\left|B_{\gamma}(a)\right|_{H}} \int_{B_{\gamma}(a)}\left|\left(b_{2}(x)-\left(b_{2}\right)_{B_{\gamma}(a)}\right) T_{k}\left(\left(b_{1}-\left(b_{1}\right)_{B_{\gamma}(a)}\right) f^{0}\right)(x)\right|^{r} d x\right)^{1 / r} \\
& +\frac{1}{\omega\left(B_{\gamma}(a)\right)}\left(\frac{1}{\left|B_{\gamma}(a)\right|_{H}} \int_{B_{\gamma}(a)}\left|T_{k}\left(\left(b_{1}-\left(b_{1}\right)_{B_{\gamma}(a)}\right)\left(b_{2}-\left(b_{2}\right)_{B_{\gamma}(a)}\right) f^{0}\right)(x)\right|^{r} d x\right)^{1 / r} \\
& +\frac{1}{\omega\left(B_{\gamma}(a)\right)} \\
& \times\left(\frac{1}{\left|B_{\gamma}(a)\right|_{H}} \int_{B_{\gamma}(a)}\left|\left(b_{1}(x)-\left(b_{1}\right)_{B_{\gamma}(a)}\right)\left(b_{2}(x)-\left(b_{2}\right)_{B_{\gamma}(a)}\right) T_{k}\left(f^{\infty}\right)(x)\right|^{r} d x\right)^{1 / r} \\
& +\frac{1}{\omega\left(B_{\gamma}(a)\right)} \\
& \times\left(\frac{1}{\left|B_{\gamma}(a)\right|_{H}} \int_{B_{\gamma}(a)}\left|\left(b_{1}(x)-\left(b_{1}\right)_{B_{\gamma}(a)}\right) T_{k}\left(\left(b_{2}-\left(b_{2}\right)_{B_{\gamma}(a)}\right) f^{\infty}\right)(x)\right|^{r} d x\right)^{1 / r} \\
& +\frac{1}{\omega\left(B_{\gamma}(a)\right)} \\
& \times\left(\frac{1}{\left|B_{\gamma}(a)\right|_{H}} \int_{B_{\gamma}(a)}\left|\left(b_{2}(x)-\left(b_{2}\right)_{B_{\gamma}(a)}\right) T_{k}\left(\left(b_{1}-\left(b_{1}\right)_{B_{\gamma}(a)}\right) f^{\infty}\right)(x)\right|^{r} d x\right)^{1 / r} \\
& +\frac{1}{\omega\left(B_{\gamma}(a)\right)}\left(\frac{1}{\left|B_{\gamma}(a)\right|_{H}} \int_{B_{\gamma}(a)}\left|T_{k}\left(\left(b_{1}-\left(b_{1}\right)_{B_{\gamma}(a)}\right)\left(b_{2}-\left(b_{2}\right)_{B_{\gamma}(a)}\right) f^{\infty}\right)(x)\right|^{r} d x\right)^{1 / r} \\
& =: E_{1}+E_{2}+E_{3}+E_{4}+E_{5}+E_{6}+E_{7}+E_{8} \text {. }
\end{aligned}
$$

We further estimate every part.

Since $1 / r=1 / q+1 / q_{1}+1 / q_{2}$, from Hölder's inequality, Lemma 2.2 , and (i) it follows that

$$
\begin{aligned}
E_{1}= & \frac{1}{\omega\left(B_{\gamma}(a)\right)} \\
& \times\left(\frac{1}{\left|B_{\gamma}(a)\right|_{H}} \int_{B_{\gamma}(a)}\left|\left(b_{1}(x)-\left(b_{1}\right)_{B_{\gamma}(a)}\right)\left(b_{2}(x)-\left(b_{2}\right)_{B_{\gamma}(a)}\right) T_{k}\left(f^{0}\right)(x)\right|^{r} d x\right)^{1 / r} \\
\leq & \frac{1}{\omega\left(B_{\gamma}(a)\right)\left|B_{\gamma}(a)\right|_{H}^{1 / r}} \prod_{i=1}^{2}\left(\int_{B_{\gamma}(a)}\left|b_{i}(x)-\left(b_{i}\right)_{B_{\gamma}(a)}\right|^{q_{i}} d x\right)^{1 / q_{i}} \\
& \times\left(\int_{B_{\gamma}(a)}\left|T_{k}\left(f^{0}\right)(x)\right|^{q} d x\right)^{1 / q}
\end{aligned}
$$


Mo et al. Journal of Inequalities and Applications

(2019) 2019:65

Page 9 of 13

$$
\begin{aligned}
& \lesssim \frac{v_{1}\left(B_{\gamma}(a)\right) \nu_{2}\left(B_{\gamma}(a)\right)}{\omega\left(B_{\gamma}(a)\right)\left|B_{\gamma}(a)\right|_{H}^{1 / q}} \prod_{i=1}^{2}\left\|b_{i}\right\|_{G C_{q_{i}, \nu_{i}}}\left(\int_{B_{\gamma}(a)}|f(x)|^{q} d x\right)^{1 / q} \\
& \leq \frac{\nu\left(B_{\gamma}(a)\right) \nu_{1}\left(B_{\gamma}(a)\right) \nu_{2}\left(B_{\gamma}(a)\right)}{\omega\left(B_{\gamma}(a)\right)} \prod_{i=1}^{2}\left\|b_{i}\right\|_{G C_{q_{i}, \nu_{i}}}\|f\|_{G M_{q, v}} \\
& \lesssim \prod_{i=1}^{2}\left\|b_{i}\right\|_{G C_{q_{i}, v_{i}}}\|f\|_{G M_{q, v} .}
\end{aligned}
$$

Let $1 / \bar{q}=1 / q+1 / q_{2}$. Then $1 / r=1 / q_{1}+1 / \bar{q}$. Thus, from Hölder's inequality, Lemma 2.2 , and (i) we obtain

$$
\begin{aligned}
& E_{2}=\frac{1}{\omega\left(B_{\gamma}(a)\right)}\left(\frac{1}{\left|B_{\gamma}(a)\right|_{H}} \int_{B_{\gamma}(a)}\left|\left(b_{1}(x)-\left(b_{1}\right)_{B_{\gamma}(a)}\right) T_{k}\left(\left(b_{2}-\left(b_{2}\right)_{B_{\gamma}(a)}\right) f^{0}\right)(x)\right|^{r} d x\right)^{1 / r} \\
& \leq \frac{1}{\omega\left(B_{\gamma}(a)\right)\left|B_{\gamma}(a)\right|_{H}^{1 / r}}\left(\int_{B_{\gamma}(a)}\left|b_{1}(x)-\left(b_{1}\right)_{B_{\gamma}(a)}\right|^{q_{1}} d x\right)^{1 / q_{1}} \\
& \times\left(\int_{B_{\gamma}(a)}\left|T_{k}\left(\left(b_{2}-\left(b_{2}\right)_{B_{\gamma}(a)}\right) f^{0}\right)(x)\right|^{\bar{q}} d x\right)^{1 / \bar{q}} \\
& \lesssim \frac{1}{\omega\left(B_{\gamma}(a)\right)\left|B_{\gamma}(a)\right|_{H}^{1 / r}}\left(\int_{B_{\gamma}(a)}\left|b_{1}(x)-\left(b_{1}\right)_{B_{\gamma}(a)}\right|^{q_{1}} d x\right)^{1 / q_{1}} \\
& \times\left(\int_{B_{\gamma}(a)}\left|\left(b_{2}(x)-\left(b_{2}\right)_{B_{\gamma}(a)}\right) f(x)\right|^{\bar{q}} d x\right)^{1 / \bar{q}} \\
& \leq \frac{1}{\omega\left(B_{\gamma}(a)\right)\left|B_{\gamma}(a)\right|_{H}^{1 / r}} \prod_{i=1}^{2}\left(\int_{B_{\gamma}(a)}\left|b_{i}(x)-\left(b_{i}\right)_{B_{\gamma}(a)}\right|^{q_{i}} d x\right)^{1 / q_{i}}\left(\int_{B_{\gamma}(a)}|f(x)|^{q} d x\right)^{1 / q} \\
& \leq \frac{v\left(B_{\gamma}(a)\right) v_{1}\left(B_{\gamma}(a)\right) v_{2}\left(B_{\gamma}(a)\right)}{\omega\left(B_{\gamma}(a)\right)} \prod_{i=1}^{2}\left\|b_{i}\right\|_{G C_{q_{i}, \nu_{i}}}\|f\|_{G M_{q, v}} \\
& \lesssim \prod_{i=1}^{2}\left\|b_{i}\right\|_{G C_{q_{i}, v_{i}}}\|f\|_{G M_{q, v}}
\end{aligned}
$$

Similarly,

$$
E_{3} \lesssim \prod_{i=1}^{2}\left\|b_{i}\right\|_{G C_{q_{i}, v_{i}}}\|f\|_{G M_{q, v}}
$$

For $E_{4}$, from Lemma 2.2, Hölder's inequality, and (i) we obtain

$$
\begin{aligned}
E_{4} & \left.=\left.\frac{1}{\omega\left(B_{\gamma}(a)\right)}\left(\frac{1}{\left|B_{\gamma}(a)\right|_{H}} \int_{B_{\gamma}(a)} \mid T_{k}\left(b_{1}-\left(b_{1}\right)_{B_{\gamma}(a)}\right)\left(b_{2}-\left(b_{2}\right)_{B_{\gamma}(a)}\right) f^{0}\right)(x)\right|^{r} d x\right)^{1 / r} \\
& \lesssim \frac{1}{\omega\left(B_{\gamma}(a)\right)\left|B_{\gamma}(a)\right|_{H}^{1 / r}}\left(\int_{B_{\gamma}(a)}\left|\left(b_{1}(x)-\left(b_{1}\right)_{B_{\gamma}(a)}\right)\left(b_{2}(x)-\left(b_{2}\right)_{B_{\gamma}(a)}\right) f(x)\right|^{r} d x\right)^{1 / r} \\
& \leq \frac{1}{\omega\left(B_{\gamma}(a)\right)\left|B_{\gamma}(a)\right|_{H}^{1 / r}} \prod_{i=1}^{2}\left(\int_{B_{\gamma}(a)}\left|b_{i}(x)-\left(b_{i}\right)_{B_{\gamma}(a)}\right|^{q_{i}} d x\right)^{1 / q_{i}}\left(\int_{B_{\gamma}(a)}|f(x)|^{q} d x\right)^{1 / q}
\end{aligned}
$$




$$
\begin{aligned}
& \leq \frac{v\left(B_{\gamma}(a)\right) v_{1}\left(B_{\gamma}(a)\right) v_{2}\left(B_{\gamma}(a)\right)}{\omega\left(B_{\gamma}(a)\right)} \prod_{i=1}^{2}\left\|b_{i}\right\|_{G C_{q_{i}, v_{i}}}\|f\|_{G M_{q, v}} \\
& \lesssim \prod_{i=1}^{2}\left\|b_{i}\right\|_{G C_{q_{i}, v_{i}}}\|f\|_{G M_{q, v}} .
\end{aligned}
$$

To estimate $E_{5}$, we first need to consider $\left|T_{k}\left(f^{\infty}\right)(x)\right|$. In fact, by (4.3) it is easy to see that

$$
\left|T_{k}\left(f^{\infty}\right)(x)\right| \lesssim\|f\|_{G M_{q, v}} \sum_{j=\gamma+1}^{\infty} v\left(B_{j}(a)\right)
$$

Therefore from Hölder's inequality, (4.6), and (ii) we get

$$
\begin{aligned}
E_{5}= & \frac{1}{\omega\left(B_{\gamma}(a)\right)} \\
& \times\left(\frac{1}{\left|B_{\gamma}(a)\right|_{H}} \int_{B_{\gamma}(a)}\left|\left(b_{1}(x)-\left(b_{1}\right)_{B_{\gamma}(a)}\right)\left(b_{2}(x)-\left(b_{2}\right)_{B_{\gamma}(a)}\right) T_{k}\left(f^{\infty}\right)(x)\right|^{r} d x\right)^{1 / r} \\
\leq & \frac{1}{\omega\left(B_{\gamma}(a)\right)\left|B_{\gamma}(a)\right|_{H}^{1 / r}} \prod_{i=1}^{2}\left(\int_{B_{\gamma}(a)}\left|b_{i}(x)-\left(b_{i}\right)_{B_{\gamma}(a)}\right|^{q_{i}} d x\right)^{1 / q_{i}} \\
& \times\left(\int_{B_{\gamma}(a)}\left|T_{k}\left(f^{\infty}\right)(x) f(x)\right|^{q} d x\right)^{1 / q} \\
\lesssim & \sum_{j=\gamma+1}^{\infty} \frac{v\left(B_{j}(a)\right) \nu_{1}\left(B_{\gamma}(a)\right) \nu_{2}\left(B_{\gamma}(a)\right)}{\omega\left(B_{\gamma}(a)\right)} \prod_{i=1}^{2}\left\|b_{i}\right\|_{G C_{q_{i}, v_{i}}}\|f\|_{G M_{q, v}} \\
\lesssim & \prod_{i=1}^{2}\left\|b_{i}\right\|_{G C_{q_{i}, v_{i}}}\|f\|_{G M_{q, v} .}
\end{aligned}
$$

It is similar to estimate (4.3) for $x \in B_{\gamma}(a)$. By $\Omega \in L^{\infty}\left(\mathbb{Q}_{p}^{n}\right)$ and (2.2) we can deduce that

$$
\begin{aligned}
\mid & \left.T_{k}\left(b_{2}-\left(b_{2}\right)_{B_{\gamma}(a)}\right) f^{\infty}\right)(x) \mid \\
& =\left|\int_{|y| p^{\prime}>p^{k}}\left(b_{2}(x-y)-\left(b_{2}\right)_{B_{\gamma}(a)}\right) f \chi_{B_{\gamma}^{c}(a)}(x-y) \frac{\Omega(y)}{|y|_{p}^{n}} d y\right| \\
& \leq \int_{B_{\gamma}^{c}}\left|b_{2}(z)-\left(b_{2}\right)_{B_{\gamma}(a)}\right||f(z)| \frac{|\Omega(x-z)|}{|x-z|_{p}^{n}} d z \\
& \lesssim \int_{B_{\gamma}^{c}} \frac{\left|b_{2}(z)-\left(b_{2}\right)_{B_{\gamma}(a)}\right||f(z)|}{|x-z|_{p}^{n}} d z \\
& \lesssim \sum_{j=\gamma+1}^{\infty} \int_{S_{j}(a)} p^{-j n}\left|b_{2}(z)-\left(b_{2}\right)_{B_{\gamma}(a)}\right||f(y)| d y \\
& =\sum_{j=\gamma+1}^{\infty} p^{-j n}\left|B_{j}(a)\right|_{H}^{1-1 / q-1 / q_{2}}\left(\int_{S_{j}(a)}|f(y)|^{q} d y\right)^{1 / q}\left(\int_{S_{j}(a)}\left|b_{2}(y)-\left(b_{2}\right)_{B_{\gamma}(a)}\right|^{q_{2}} d y\right)^{1 / q_{2}}
\end{aligned}
$$




$$
\begin{aligned}
& \leq\|f\|_{G M_{q, v}} \sum_{j=\gamma+1}^{\infty} p^{-j n}\left|B_{j}(a)\right|_{H}^{1-1 / q_{2}} v\left(B_{j}(a)\right)\left(\int_{B_{j}(a)}\left|b_{2}(y)-\left(b_{2}\right)_{B_{\gamma}(a)}\right|^{q_{2}} d y\right)^{1 / q_{2}} \\
& \lesssim\left\|b_{2}\right\|_{G C_{q_{2}, v_{2}}}\|f\|_{G M_{q, v}} \sum_{j=\gamma+1}^{\infty}(j+1-\gamma) v\left(B_{j}(a)\right) v_{2}\left(B_{j}(a)\right) .
\end{aligned}
$$

Let $1 / \bar{q}=1 / q+1 / q_{2}$. Then $1 / r=1 / q_{1}+1 / \bar{q}$. Thus from Hölder's inequality, (4.7), and (ii) it follows that

$$
\begin{aligned}
E_{6}= & \frac{1}{\omega\left(B_{\gamma}(a)\right)}\left(\frac{1}{\left|B_{\gamma}(a)\right|_{H}} \int_{B_{\gamma}(a)} \mid\left(b_{1}(x)-\left(b_{1}\right)_{B_{\gamma}(a)}\right) T_{k}\left(\left.\left(b_{2}-\left(b_{2}\right)_{\left.B_{\gamma}(a)\right)} f^{\infty}\right)(x)\right|^{r} d x\right)^{1 / r}\right. \\
\leq & \frac{1}{\omega\left(B_{\gamma}(a)\right)\left|B_{\gamma}(a)\right|_{H}^{1 / r}}\left(\int_{B_{\gamma}(a)}\left|b_{1}(x)-\left(b_{1}\right)_{B_{\gamma}(a)}\right|^{q_{1}} d x\right)^{1 / q_{1}} \\
& \times\left(\int_{B_{\gamma}(a)}\left|T_{k}\left(\left(b_{2}-\left(b_{2}\right)_{B_{\gamma}(a)}\right) f^{\infty}\right)(x)\right|^{\bar{q}} d x\right)^{1 / \bar{q}} \\
\leq & \prod_{i=1}^{2}\left\|b_{i}\right\|_{G C_{q_{i}, v_{i}}}\|f\|_{G M_{q, v}} \frac{1}{\omega\left(B_{\gamma}(a)\right)} \sum_{j=\gamma+1}^{\infty}(j+1-\gamma) v\left(B_{j}(a)\right) \nu_{2}\left(B_{j}(a)\right) \nu_{1}\left(B_{\gamma}(a)\right) \\
\lesssim & \prod_{i=1}^{2}\left\|b_{i}\right\|_{G C_{q_{i}, v_{i}}}\|f\|_{G M_{q, v}} .
\end{aligned}
$$

Similarly estimating $E_{6}$, we obtain

$$
E_{7} \lesssim \prod_{i=1}^{2}\left\|b_{i}\right\|_{G C_{q_{i}, v_{i}}}\|f\|_{G M_{q, v}} .
$$

Moreover, since $\Omega \in L^{\infty}\left(\mathbb{Q}_{p}^{n}\right)$, by (2.2) it is easy to see that

$$
\begin{aligned}
\mid T_{k} & \left(\left(b_{1}-\left(b_{1}\right)_{B_{\gamma}(a)}\right)\left(b_{2}-\left(b_{2}\right)_{B_{\gamma}(a)}\right) f^{\infty}\right)(x) \mid \\
= & \left|\int_{|x-z|_{p}>p^{k}}\left(b_{1}(z)-\left(b_{1}\right)_{B_{\gamma}(a)}\right)\left(b_{2}(z)-\left(b_{2}\right)_{B_{\gamma}(a)}\right) f \chi_{B_{\gamma}^{c}(a)}(z) \frac{\Omega(x-z)}{|x-z|_{p}^{n}} d z\right| \\
\leq & \int_{B_{\gamma}^{c}}\left|b_{1}(z)-\left(b_{1}\right)_{B_{\gamma}(a)}\right|\left|b_{2}(z)-\left(b_{2}\right)_{B_{\gamma}(a)}\right| f(z) \mid \frac{|\Omega(x-z)|}{|x-z|_{p}^{n}} d z \\
\lesssim & \sum_{j=\gamma+1}^{\infty} \int_{S_{j}(a)} p^{-j n}\left|b_{1}(z)-\left(b_{1}\right)_{B_{\gamma}(a)}\right|\left|b_{2}(z)-\left(b_{2}\right)_{B_{\gamma}(a)}\right||f(y)| d y \\
= & \sum_{j=\gamma+1}^{\infty} p^{-j n}\left|B_{j}(a)\right|_{H}^{1-1 / q-1 / q_{1}-1 / q_{2}}\left(\int_{S_{j}(a)}|f(y)|^{q} d y\right)^{1 / q} \\
& \times\left(\int_{S_{j}(a)}\left|b_{1}(y)-\left(b_{1}\right)_{B_{\gamma}(a)}\right|^{q_{1}} d y\right)^{1 / q_{1}} \\
& \times\left(\int_{S_{j}(a)}\left|b_{2}(y)-\left(b_{2}\right)_{B_{\gamma}(a)}\right|^{q_{2}} d y\right)^{1 / q_{2}} \\
& \lesssim \prod_{i=1}^{2}\left\|b_{i}\right\|_{G C_{q_{i}, v_{i}}}|| f \|_{G M_{q, v}} \sum_{j=\gamma+1}^{\infty}(j+1-\gamma)^{2} v\left(B_{j}(a)\right) \nu_{1}\left(B_{j}(a)\right) \nu_{2}\left(B_{j}(a)\right) .
\end{aligned}
$$


Therefore from (4.8) and (ii) we get that

$$
\begin{aligned}
E_{8} & =\frac{1}{\omega\left(B_{\gamma}(a)\right)}\left(\frac{1}{\left.B_{\gamma}(a)\right|_{H}} \int_{B}\left|T_{k}\left(\left(b_{1}-\left(b_{1}\right)_{B_{\gamma}(a)}\right)\left(b_{2}-\left(b_{2}\right)_{B_{\gamma}(a)}\right) f^{\infty}\right)(x)\right|^{r} d x\right)^{1 / r} \\
& \leq \prod_{i=1}^{2}\left\|b_{i}\right\|_{G C_{q_{i}, v_{i}}}\|f\|_{G M_{q, v}} \frac{1}{\omega\left(B_{\gamma}(a)\right)} \sum_{j=\gamma+1}^{\infty}(j+1-\gamma)^{2} v\left(B_{j}(a)\right) \nu_{1}\left(B_{j}(a)\right) \nu_{2}\left(B_{j}(a)\right) \\
& \lesssim \prod_{i=1}^{2}\left\|b_{i}\right\|_{G C_{q_{i}, v_{i}}}\|f\|_{G M_{q, v}} .
\end{aligned}
$$

Combining (4.5) and the estimates of $E_{1}, E_{2}, \ldots, E_{8}$, we have

$$
\frac{1}{\omega\left(B_{\gamma}(a)\right)}\left(\frac{1}{\left|B_{\gamma}(a)\right|_{H}} \int_{B_{\gamma}(a)}\left|T_{k}^{\left(b_{1}, b_{2}\right)}(f)(x)\right|^{r} d x\right)^{1 / r} \leq \prod_{i=1}^{2}\left\|b_{i}\right\|_{G C_{q_{i}, v_{i}}}\|f\|_{G M_{q, v}},
$$

which means that the commutator $T_{k}^{\left(b_{1}, b_{2}\right)}$ is bounded from $G M_{q, v}$ to $G M_{r, \omega}$.

Moreover, by Lemma 2.2 and the definition of $G M_{q, \omega}\left(\mathbb{Q}_{p}^{n}\right)$ it is obvious that the commutator $T^{\vec{b}}(f)=\lim _{k \rightarrow-\infty} T_{k}^{\vec{b}}(f)$ exists in the space of $G M_{q, \omega}$, and $T^{\vec{b}}$ is bounded from $G M_{q, v}$ to $G M_{q, \omega}$.

Therefore the proof of Theorem 3.3 is complete.

\section{Conclusion}

In this paper, we established the boundedness of a class of $p$-adic singular integral operators on the $p$-adic generalized Morrey spaces. We also considered the corresponding boundedness for the commutators generalized by the $p$-adic singular integral operators and $p$-adic Lipschitz functions or $p$-adic generalized Campanato functions.

\section{Acknowledgements}

The authors are grateful to the editor and referees for carefully reading the manuscript.

Funding

The work is supported by National Natural Science Foundation of China (No. 11601035).

Availability of data and materials

No data were used to support this study.

Competing interests

The authors declare that they have no competing interests.

Authors' contributions

All authors have made equal contributions in this article. All authors read and approved the final manuscript.

\section{Publisher's Note}

Springer Nature remains neutral with regard to jurisdictional claims in published maps and institutional affiliations.

Received: 18 December 2018 Accepted: 25 February 2019 Published online: 13 March 2019

\section{References}

1. Vladimirov, V.S., Volovich, I.V., Zelenov, E.I.: P-adic Analysis and Mathematical Physics. World Scientific, Singapore (1994)

2. Schikhof, W.H.: Ultrametric Calculus: An Introduction to p-Adic Analysis, vol. 4. Cambridge University Press, London (2007)

3. Robert, A.M.: A Course in p-Adic Analysis, vol. 198. Springer, New York (2013)

4. Chuong, N.M., Egorov, Y.V., Khrennikov, A., Meyer, Y., Mumford, D.: Harmonic, Wavelet and p-Adic Analysis. World Scientific, Singapore (2007) 
5. Taibleson, M.H.: Fourier Analysis on Local Fields. Princeton University Press, Princeton (1975)

6. Haran, S.: Analytic potential theory over the $p$-adics. Ann. Inst. Fourier 43(3), 905-944 (1993)

7. Kim, Y.C.: A simple proof of the $p$-adic version of the Sobolev embedding theorem. Commun. Korean Math. Soc $25(1), 27-36(2010)$

8. Wu, Q.Y., Fu, Z.W.: Hardy-Littlewood-Sobolev inequalities on p-adic central Morrey spaces. J. Funct. Spaces 2015, Article ID 419532 (2015)

9. Albeverio, S., Karwoski, W.: A random walk on p-adics: the generator and its spectrum. Stoch. Process. Appl. 53, 1-22 (1944)

10. Avetisov, A.V., Bikulov, A.H., Kozyrev, S.V., Osipov, V.A.: -adic models of ultrametric diffusion constrained by hierarchical energy landscapes. J. Phys. A, Math. Gen. 35, 177-189 (2002)

11. Phillips, K., Taibleson, M.: Singular integrals in several variables over a local field. Pac. J. Math. 30, 209-231 (1969)

12. Wu, Q.Y., Lu, S.Z., Fu, Z.W.: $p$-adic central function spaces and singular integral operators. Chin. J. Contemp. Math. 37(4), 1-18 (2016)

13. Volosivets, S.S.: Maximal function and Riesz potential on $p$-adic linear spaces. $p$-adic numbers ultrametric. Anal. Appl. 5(3), 226-234 (2013)

14. Mo, H., Wang, X., Ma, R.: Commutator of Riesz potential in p-adic generalized Morrey spaces. Front. Math. China 13(3), 633-645 (2018)

15. Nakai, E.: Hardy-Littlewood maximal operator, singular integral operators and the Riesz potentials on generalized Morrey spaces. Math. Nachr. 166, 95-103 (1994)

16. Morrey, C.: On the solutions of quasi-linear elliptic partial differential equations. Trans. Am. Math. Soc. 43, 126-166 (1938)

17. Chuong, N.M., Duong, D.V.: Weighted Hardy-Llittlewood operators and commutators on $p$-adic functional spaces. P-adic numbers ultrametric. Anal. Appl. 5(1), 65-82 (2013)

18. Campanato, S.: Proprietà di Hölderianità di alcune classi di funzioni. Ann. Sc. Norm. Super. Pisa 17, 175-188 (1963)

19. Peetre, J.: On the theory of $p, \lambda$ spaces. J. Funct. Anal. 4, 71-87 (1969)

\section{Submit your manuscript to a SpringerOpen ${ }^{\circ}$ journal and benefit from:}

- Convenient online submission

- Rigorous peer review

- Open access: articles freely available online

- High visibility within the field

- Retaining the copyright to your article

Submit your next manuscript at $\gg$ springeropen.com 